\title{
The Field Confinement, Narrow Transmission Resonances, and Green Function of a Multilayered Microsphere with Metamaterial Defects
}

\author{
Gennadiy Burlak and A. Díaz-de-Anda \\ Centro de Investigación en Ingeniería y Ciencias Aplicadas, Universidad Autónoma del Estado de Morelos, \\ 62209 Cuernavaca, MOR, Mexico \\ Correspondence should be addressed to Gennadiy Burlak, gburlak@uaem.mx
}

Received 30 June 2011; Revised 3 August 2011; Accepted 6 August 2011

Academic Editor: Alan Migdall

Copyright (C 2011 G. Burlak and A. Díaz-de-Anda. This is an open access article distributed under the Creative Commons Attribution License, which permits unrestricted use, distribution, and reproduction in any medium, provided the original work is properly cited.

\begin{abstract}
We numerically investigate the optical transmission through a compound spherical stack with conventional and metamaterial (MM) layers and also embedded MM defect. A formation of extremely narrow resonant peak with nearly complete transmittance in area of a band gap is found. We demonstrate that photon fields of certain frequencies can be strongly confined by a left-handed (LH) defect. The influence of a random deviation in the width of compound spherical layers as well the transit to the whispering gallery mode (WGM) is also discussed.
\end{abstract}

\section{Introduction}

Controlling the electromagnetic properties of materials, going beyond the limit that is attainable with naturally existing substances, has become a reality with the advent of metamaterials $[1,2]$. The range of various structured artificial structures has promised a vast variety of otherwise unexpected physical phenomena [3-5] among which the experimental realization of a negative refractive index has been one of the main advantage.

A typical metamaterial combines continuous metal films (about $50 \mathrm{~nm}$ ) with nanostrip magnetic resonators that finally yield a negative-index material. The film can be formed with a mixture of dielectric (e.g., silica) and metal, such as silver or gold, forming a semicontinuous metal film. Such structures are fabricated using techniques as the evaporation of a metal onto a dielectric substrate (see more details in review [5]). Preparation of metamaterial structure in the optical range still is an advanced task. In recent experiment [6] it has been demonstrated that the incorporation of the gain material (rhodamine 800) in the metamaterial makes it possible to fabricate an extremely low-loss and an active optical negative-index metamaterial (NIM) that is not limited by the inherent loss in its metal constituent. In this experiment the optical (NIM) structure (silver layers + rhodamine 800) is the fishnet with periodicity about $300 \mathrm{~nm}$. Epoxy doped with rhodamine 800 (Rh800) and dye is used as a gain medium. The loss compensation mechanism in the sample is straightforward to understand. When Rh800 is excited by a pump pulse with sufficiently high power, a population inversion is formed inside the dye molecules. This provides amplification for a properly delayed probe pulse whose wavelength is coincident with the stimulated emission wavelength of the dye molecules (see details in [6]).

Natural extension of such directions is the analysis of a compacted spherical multilayered system with embedded metamaterial layers. It is well known that except for the whispering gallery mode (WGM) regime [7-10], a bare dielectric sphere has a complex spectrum of the electromagnetic low-quality ( $Q$ factor) eigenoscillations because of the energy leakage into the outer space [11]. The case of the compound structure, when the dielectric sphere is coated by an alternative stack, is much richer. The $Q$-factor of optical 
oscillations has a large value in the frequency regions of weak transmittance and, beyond these regions $Q$, remains small [12-14]. This gives rise to a large variety of optical properties of microspheres coated with a multilayer stack. Such a system can serve as a spherical symmetric photonic band gap structure, which possesses strong selective transmittance properties $[15,16]$, with the incorporated nanometer-sized photon emitters. These possibilities essentially allow the expansion of the operational properties of microspheres with attractive artificial light sources for advanced optical technologies. The energy confinement into small volumes has applications in many fields such as photonics and quantum electrodynamics. In this sense, the microstructures that have the greatest ability to store energy for long periods of time are the dielectric spherical resonators. Recently, it has been feasible to construct such a microsphere accurately, and the parameters may be precisely controlled and measured [17]. New materials are used to extend the optical properties of layered microspheres [18].

In studying such an effect in multilayered microspheres, the following question emerges: how does such an effect will be changed at a deviation from strict periodicity of a stack? Generally such a case can be interpreted as a translation symmetry breaking due to placing of defect layers in the periodic stack. The question rises whether it is possible to obtain narrow transmittance peaks in an alternating spherical stack with inserting a defect layer?

A translation symmetry breaking can be achieved in a quasiperiodic system. In [19-21] optical properties of layered microsphere with a dielectric stack, in which optical layers are constructed following the Fibonacci sequence, were investigated. Such a quasiperiodic structure serves as an intermediate case between a strictly periodic and cleanly casual sequence of alternative layers in a stack. It was found that when the number of layers (Fibonacci order) increases, the structure of the spectrum acquires a fractal form. If the metamaterial layers (with negative refraction index) are included in such a stack then the width of the resonant peaks in the frequency spectrum becomes extremely narrow [22]. It is found the formation and coupling of defect modes in two-dimensional photonic crystal (PC) band gaps associated with degenerate edges [23]. In very recent article [24] it has been shown that by varying the radii and permittivity of the defects, total transmission or reflection of the impinging electromagnetic wave can be achieved.

In this paper, we study the photon transmissions through the compound spherical stack containing conventional and metamaterial (MM) layers and also an embedded MM defect that are transparent and can bend light in opposite directions. A narrow well-separated resonant peak with nearly complete transmittance in a bandgap arises when such a MM defect layer is embedded to the center of the spherical stack. We demonstrate that photon fields of certain frequencies can be strongly confined by a left-handed (LH) defect. The influence of a random deviation in the width of compound spherical layers as well the transit to whisperinggallery mode (WGM) is also discussed.

\section{Basic Equation}

The geometry of the multilayered microsphere with the embedded defect is depicted in Figure 1.

Despite that the peculiarities of the propagation of optical waves in $1 \mathrm{D}$ plane systems with defects are investigated in a number of articles the results of such studies cannot be applied directly to a spherical stack for the following reasons. (i) In a spherical case, the transfer matrices $\underline{M}_{j}$ have the determinant depending on number $j$ of a layer as $\operatorname{det}\left(\underline{M}_{j}\right)=\left(r_{j+1} / r_{j}\right)^{2}$ [14] (is not unimodular), where $r_{i+1}$ and $r_{j}$ are radii of the external and internal boundaries of the layer, respectively. Such a structure of the transfer matrix is physically caused by preservation of the energy flux in a radial direction in a solid spherical angle. (ii) In a spherical stack, the transfer matrix $\underline{M}_{j}$ is quite involved since it is written through the complex Hankel functions. Physically, it is due to the preferential role of the pole (center) of a microsphere that breaks the translational symmetry in such a system. Furthermore, in a spherical geometry, the transmittance coefficient $T$ depends on the spherical quantum number $m$ (angular momentum), for great $m \gg 1$ that leads to a whispering gallery mode (WGM) regime with practically zero transmittance in a frequency range.

In order to study the optical properties of a spherical stack with embedded defect layer, let us first formulate the transfer matrix method exploited in spherical multilayered geometry. Various approaches were proposed [14, 25-28] (see $[29,30]$ and references therein). Here we follow [25].

In a multilayered microsphere, the set of the Maxwell equation is

$$
\nabla \times \vec{H}=i \omega \varepsilon_{0} \varepsilon(\omega) \vec{E}, \quad \nabla \times \vec{E}=-i \omega \vec{B},
$$

where $\vec{E}$ and $\vec{B}$ are electric and magnetic fields and $\varepsilon(\omega)$ is a dielectric permittivity of a layer. We use the complex exponential multiplier in the form $\exp (i \omega t)$. Equation (1) in the spherical coordinate frame $(r, \theta, \varphi)$ usually reduces to the Helmholtz equation for a scalar function called the Debye potential $\Pi(r, \theta, \varphi)$ [31]. Since our calculations did not register significant difference between TM and TE wave cases further we will concentrate mainly in the TM case. The equation for the radial part of the Debye-potential $\Pi=\Pi(r)$ in a layer can be readily obtained from (1) and is given by

$$
\frac{d^{2} \Pi}{d r^{2}}+\left[\varepsilon(\omega) k_{0}^{2}-\frac{l(l+1)}{r^{2}}\right] \Pi=0,
$$

where $k_{0}=\omega / c$. Equation (2) is easily solved in terms of spherical Hankel functions. In each layer of the stack, we use the next matrix presentation for the fields

$$
\vec{u}=\left[\begin{array}{c}
H_{\phi} \\
E_{\theta}
\end{array}\right]=\underline{D} \cdot\left[\begin{array}{l}
a \\
b
\end{array}\right]=\underline{D} \cdot \vec{q}, \quad \vec{q}=\left[\begin{array}{l}
a \\
b
\end{array}\right],
$$

where $a$ and $b$ are arbitrary constants, and matrix $\underline{D}=\underline{D}(y)$ is given by

$$
\underline{D}=\left[\begin{array}{cc}
i n P_{m}^{(2)}(y) e^{i y} & i n P_{m}^{(1)}(y) e^{-i y} \\
G_{m}^{(2)}(y) e^{i y} & G_{m}^{(1)}(y) e^{-i y}
\end{array}\right] .
$$




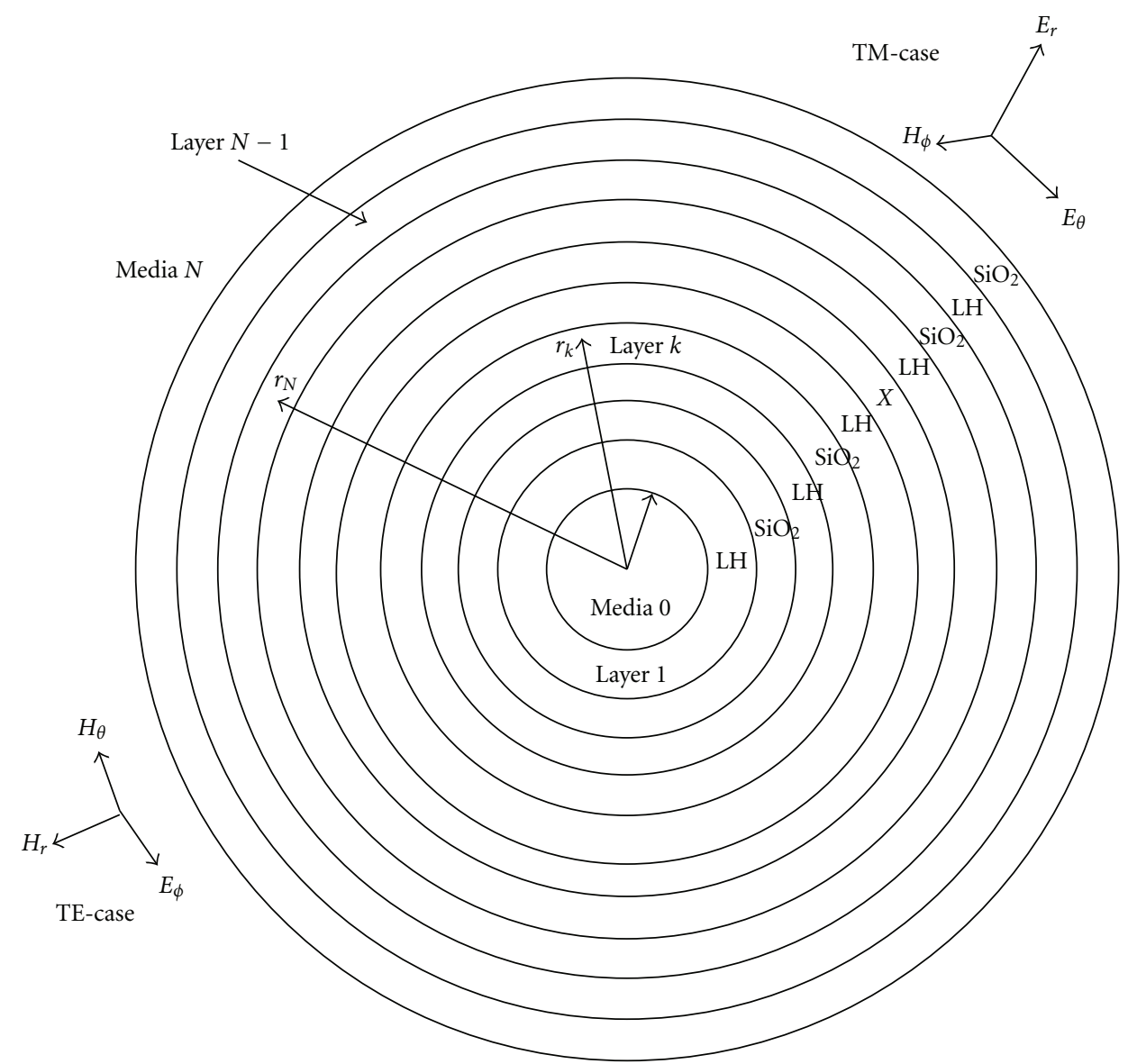

FIGURE 1: Geometry of the system. Multilayered microsphere $\left(\mathrm{LH}+\mathrm{SiO}_{2}\right)$ with a defect $\mathrm{LH}$ layer (indicated as $\left.X\right)$ in a spherical stack.

Here, $n=n(\omega)=\sqrt{\varepsilon(\omega)}$ is the refractive index of a particular layer (that can be positive or negative for metamaterial layers); $P_{m}^{(1,2)}(y)$ is the rational part of Hankel spherical functions $h_{m}^{(1,2)}(y)=P_{m}^{(1,2)}(y) e^{ \pm i y} ; G_{m}^{(1,2)}(y)$ is the rational part of derivative of Hankel spherical functions $(\partial / \partial y) h_{m}^{(1,2)}(y)=G_{m}^{(1,2)}(y) e^{ \pm i y} ; m$ is the number of a spherical harmonic, $y=\omega n(\omega) r / c$. The recursive relations for calculations of $P_{m}^{(1,2)}(y)$ and $G_{m}^{(1,2)}(y)$ are given in [26]. Below, we provide the matrix $\underline{D}$, as well as vectors $\vec{q}$ and $\vec{u}$ by indices according to the number of layers in the stack. As the vector $\vec{q}$ in each layer is constant, for any two points $r_{1}$ and $r_{2}$ of a $k$-layer we obtain from (3) the following:

$$
\vec{q}_{k}=\underline{D}_{k}^{-1}\left(r_{1}\right) \cdot \vec{u}_{k}\left(r_{1}\right)=\underline{D}_{k}^{-1}\left(r_{2}\right) \cdot \vec{u}_{k}\left(r_{2}\right)
$$

To start the calculation, we assume that the points $r_{1}$ and $r_{2}$ belong to the boundaries of layers; $r_{2}=r_{1}+d_{1}, d_{1}$ is the thickness of the layer. At the boundary between layers, $k$ and $k+1$, the continuity of fields gives $\overrightarrow{\mathcal{u}}_{k}\left(r_{2}\right)=\overrightarrow{\mathcal{u}}_{k+1}\left(r_{2}\right)$. With the latter (5) can be rewritten as

$$
\vec{u}_{k}\left(r_{1}\right)=\underline{D}_{k}\left(r_{1}\right) \cdot \underline{D}_{k}^{-1}\left(r_{2}\right) \cdot \vec{u}_{k+1}\left(r_{2}\right) \equiv \underline{M}_{k} \cdot \vec{u}_{k+1}\left(r_{2}\right) .
$$

This relation can be extended as follows. Let us start from the bottom layer of the stack with the number $k=1$. Then from (6) we have

$$
\begin{aligned}
\vec{u}_{1} & \equiv \vec{u}_{1}\left(r_{1}\right)=\underline{M}_{1} \cdot \vec{u}_{2}=\underline{M}_{1} \cdot \underline{M}_{2} \cdot \vec{u}_{3} \\
& =\underline{M}_{1} \cdot \underline{M}_{2} \ldots \cdot \underline{M}_{N-1} \cdot \vec{u}_{N} \equiv \underline{M} \cdot \vec{u}_{N}=\underline{M} \cdot \vec{u}_{N}\left(r_{N}\right),
\end{aligned}
$$

where

$$
\underline{M}=\prod_{k=1}^{N-1} \underline{M}_{k}
$$

is the transfer matrix between inner and outer layers in the spherical stack.

In the simplest case of the spherical mode $m=1$, the elements of matrix $\underline{M}_{k}$ in (8) are

$$
\begin{aligned}
\underline{M}_{11}= & \frac{\left(y_{2}-y_{1}+y_{1} y_{2}^{2}\right) \cos \left(\Phi_{k}\right)}{y_{1}^{2} y_{2}} \\
& +\frac{\left(-1+y_{2}^{2}-y_{1} y_{2}\right) \sin \left(\Phi_{k}\right)}{y_{1}^{2} y_{2}},
\end{aligned}
$$




$$
\begin{aligned}
\underline{M}_{12}= & n_{k} \frac{i\left(y_{2}-y_{1}\right) \cos \left(\Phi_{k}\right)}{y_{1}^{2}}-n_{k} \frac{i\left(1+y_{2} y_{1}\right) \sin \left(\Phi_{k}\right)}{y_{1}^{2}}, \\
\underline{M}_{21}= & \frac{-i\left(1+y_{2} y_{1}\right)\left(-y_{2}+y_{1}\right) \cos \Phi_{1}}{y_{2} y_{1}^{3} n_{k}}, \\
& -i \frac{\left(1-y_{2}^{2}+y_{2} y_{1}-y_{1}^{2}+y_{1}^{2} y_{2}^{2}\right) \sin \left(\Phi_{k}\right)}{y_{1}^{3} y_{2} n_{k}}, \\
\underline{M}_{22}= & \frac{\left(-y_{2}+y_{1}+y_{1}^{2} y_{2}\right) \cos \left(\Phi_{k}\right)}{y_{1}^{3}} \\
& +\frac{\left(1+y_{1} y_{2}-y_{1}^{2}\right) \sin \left(\Phi_{k}\right)}{y_{1}^{3}},
\end{aligned}
$$

where $y_{1} \equiv y_{k, k}, y_{2} \equiv y_{k, k+1}, y_{k, l}=k_{0} n_{k} r_{l}, y_{2}-y_{1}=$ $\Phi_{k}=k_{0} n_{k} d_{k} ; d_{k}$ is the thickness of the $k$ th layer. From (9), one can see that in the spherical case, the transition matrix $\underline{M}_{k}$ depends explicitly on the distance to the center of the microsphere.

Note that in the case where $r_{k} \gg d_{k}$ or $y_{1} \sim y_{2} \gg 1$ in (8), one gets the well-known plane case expression for $\underline{M}_{k}$ [32]:

$$
\underline{M}_{k}=\left[\begin{array}{cc}
\cos \Phi_{k} & -i n_{k} \sin \Phi_{k} \\
-i \frac{1}{n_{k}} \sin \Phi_{k} & \cos \Phi_{k}
\end{array}\right] .
$$

Only if such an approximation is fulfilled, the matrix elements in (10) are independent of the absolute values of the coordinates and dependent only on the thicknesses of layers $d_{k}$ through value $y_{2}-y_{1}=\Phi_{k}$. This means that in general, the local properties of electromagnetic oscillations depend on the place of a layer with respect to the center of the spherical cavity.

Equation (7) connects the values of the fields on both external and internal boundaries of the spherical stack. Therefore, solving the Maxwell equations is completely equivalent to calculating products of the transfer matrices $\underline{M}$. We have used the Sommerfeld radiation conditions, where there is only an outgoing wave at the external boundary that gives $\vec{q}_{N}$ in (3) $b_{N}=0$. As a result, the amplitudes of waves in the internal substrate become

$$
\vec{u}_{0}=a_{0} \cdot\left[\begin{array}{l}
1 \\
R
\end{array}\right]=\underline{D}_{0}^{-1} \cdot \underline{M} \cdot \underline{D}_{N} \cdot \vec{q}_{N}=a_{0} \sigma \cdot \underline{Q} \cdot\left[\begin{array}{l}
T \\
0
\end{array}\right],
$$

where the matrix $Q=\underline{D}_{0}^{-1} \cdot \underline{M} \cdot \underline{D}_{N}, R=b_{0} / a_{0}$ is a reflection coefficient; $T=\overline{a_{N}} / \sigma a_{0}$ is a transmittance coefficient; $\sigma=$ $\left(n_{0} / n_{N}\right)^{1 / 2}$. After solving (11), one can easily obtain $R$ and $T$ in the next form:

$$
R=\frac{\underline{Q}_{21}(\omega)}{\underline{Q}_{11}(\omega)}, \quad T=\frac{1}{\sigma \underline{Q}_{11}(\omega)} .
$$

In (12), two equations interrelate three variables: $R, T$, and frequency $\omega$. Defining $\omega$, one can calculate the frequency dependence of $R(\omega), T(\omega)$ for the stack.
We can use (3)-(12) to calculate the reflectance, transmittance, eigenfrequencies, and eigenfields in an arbitrary spherical stack. Since no specific properties of a spherical stack were used, one can conclude that (3)-(11) are valid for any structure of the spherical stack. Now, we exploit such a technique for studying a combined alternating spherical stack with conventional and metamaterial layers.

Further we consider metamaterial layers characterized by a (relative) permittivity $\varepsilon(\mathbf{r}, \omega)$ and a (relative) permeability $\mu(\mathbf{r}, \omega)$, both of which are spatially varying, complex functions of frequency satisfying the relations $[33,34]$

$$
n(\mathbf{r}, \omega)=\sqrt{|\varepsilon(\mathbf{r}, \omega) \mu(\mathbf{r}, \omega)|} e^{i\left[\phi_{\varepsilon}(\mathbf{r}, \omega)+\phi_{\mu}(\mathbf{r}, \omega)\right] / 2} .
$$

In the following, we refer to the material of a layer as being left handed ( $\mathrm{LH}$ or metamaterial) if the real part of its refractive index is negative. In order to allow a dependence on the frequency of the refractive index, let us restrict our attention to a single-resonance permittivity

$$
\varepsilon(\omega)=1+\frac{\omega_{P e}^{2}}{\omega_{T e}^{2}-\omega^{2}-i \omega \gamma_{e}}
$$

and a single-resonance permeability

$$
\mu(\omega)=1+\frac{\omega_{P m}^{2}}{\omega_{T m}^{2}-\omega^{2}-i \omega \gamma_{m}},
$$

where $\omega_{P e}, \omega_{P m}$ are the coupling strengths, $\omega_{T e}, \omega_{T m}$ are the transverse resonance frequencies, and $\gamma_{e}, \gamma_{m}$ are the absorption parameters. In our case we used the following typical values: $\omega_{T m} / 2 \pi=f_{T m}=159.2 \mathrm{THz}, \gamma_{m} / 2 \pi=f \gamma=$ $0.001592 \mathrm{THz}, f_{T e}=163.9 \mathrm{THz}, f_{P e}=119.4 \mathrm{THz}, f_{P m}=$ $68.44 \mathrm{THz}$.

In the optical range the performance of all NIM applications is significantly limited by the inherent and strong energy dissipation in metals, especially in the near-infrared and visible wavelength ranges [5]. In our study we have explored mainly the case where the loss compensation can be attained. It is worth noting that in general case both dependencies $\varepsilon(\omega)$ and $\mu(\omega)$ may be more complicated than used in (14) and (15). However, since the metamaterials are artificial structures there are no strict expressions for $\varepsilon(\omega)$ and $\mu(\omega)$ derived from the first principles up to now. Some analytical approximations were obtained numerically in order to fit the experiment data smoothly. In this paper we use a single (or double) resonance structure for $\varepsilon(\omega)$ and $\mu(\omega)$ similarly that was detected in [6, Figure 4(b)]. On the other hand we use the relations (14)-(15) that already were used in a number of articles $[33,35,36]$ and are in agreement with the principle of causality as expressed in the Kramers-Kronig relations (see recent discussion in $[37,38]$ ). We believe that such an approach cannot lead to significant errors. This allows the use in (14)-(15) of the dissipation parameter $\gamma$ with small value $\gamma / 2 \pi=0.0016 \mathrm{THz}$.

Figure 2(b) shows the LH refractive index $n(\mathbf{r}, \omega)=$ $\operatorname{Re} n(\mathbf{r}, \omega)+i \operatorname{Im} n(\mathbf{r}, \omega)(\omega=2 \pi f$, with the permittivity $\varepsilon(\omega)$ and the permeability $\mu(\omega)$ being, resp., given by (14) and (15)). In the inset, the details of $n(\mathbf{r}, \omega)$ are shown in the 


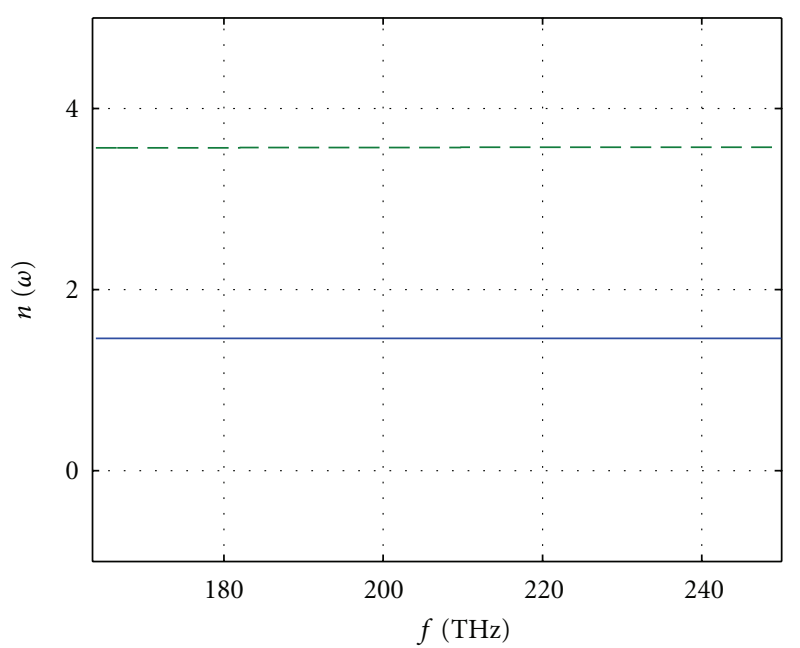

(a)

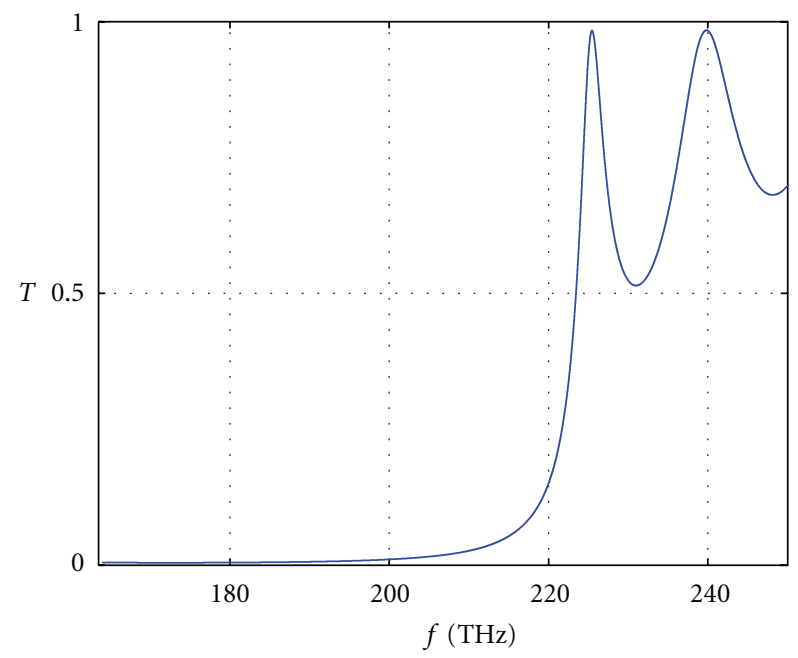

(c)

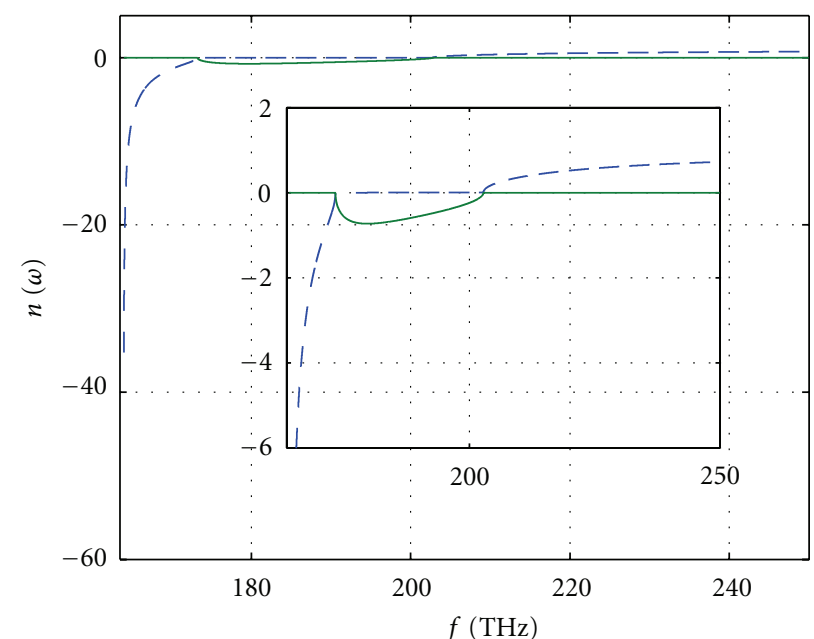

(b)

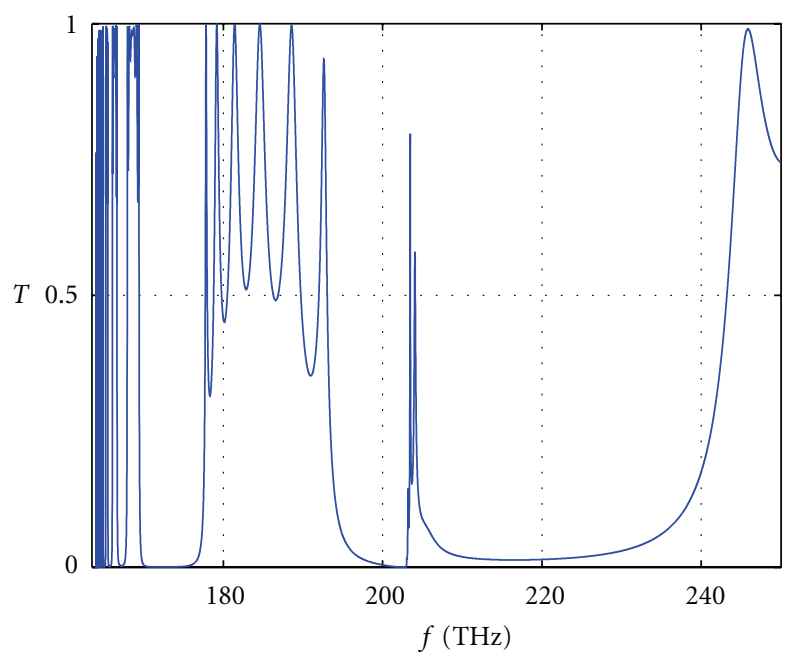

(d)

Figure 2: (Color online) Comparison of the spectral properties of the transmission coefficients $T$ for 15-layered alternating stacks containing conventional $\left(\mathrm{Si}+\mathrm{SiO}_{2}\right)$ and $\left(\mathrm{LH}+\mathrm{SiO}_{2}\right)$ materials correspondingly (spherical number $\left.m=1\right):($ a) the refractive index $n(\omega)(f=\omega / 2 \pi)$ for the $\mathrm{Si}$ (dotted line) and $\mathrm{SiO}_{2}$ (solid line); (b) the real (dotted line) and imaginary part (solid line) of the refraction coefficient $n(\omega)$ for LH metamaterial. The spectrum $T(\omega)$ is shown in (c) for conventional stack, while (d) shows $T(\omega)$ for a combined stack when Si is replaced with LH layers. See details in text.

frequency interval from $164 \mathrm{THz}$ where $\operatorname{Re} n(\mathbf{r}, \omega)<0$. It is worth noting that the negative real part of the refractive index is typically observed together with strong dispersion, so that absorption cannot be disregarded in general. However, in a very recent work [6], it was experimentally demonstrated that the incorporation of gain material in a metamaterial makes it possible to fabricate extremely low-loss and active optical devices. Thus, the original loss-limited negative refractive index can be drastically improved with the loss compensation in the visible wavelength range.

It is worth noting that the features of the use of tensorial or scalar $\varepsilon(\omega)$ and $\mu(\omega)$ expressions for metamaterials in spherical geometry (as well for other types of curved boundaries) up to now are an open problem. Synthesized technologies are mainly elaborated for preparation of hybrid spherical layered structures (see [18] and references therein). To the best of our knowledge the separation of component $\varepsilon(\omega)$ and $\mu(\omega)$ for spherical interfaces is not studied in details. However, as far as the ratio of typical sizes of the metamaterial structures (period of fishnet) and microsphere is small (about 0.1 an less) and to seek for simplicity we use the average values $\varepsilon(\omega)$ and $\mu(\omega)$ that are scalar in the first approximation.

\section{Numerical Results}

Our numerical results are shown in Figures (2)-(10). The following parameters have been used in our calculations: the geometry of system is $A,\{B, C\}, \ldots, G, \ldots\{B, C\}, D$, where 


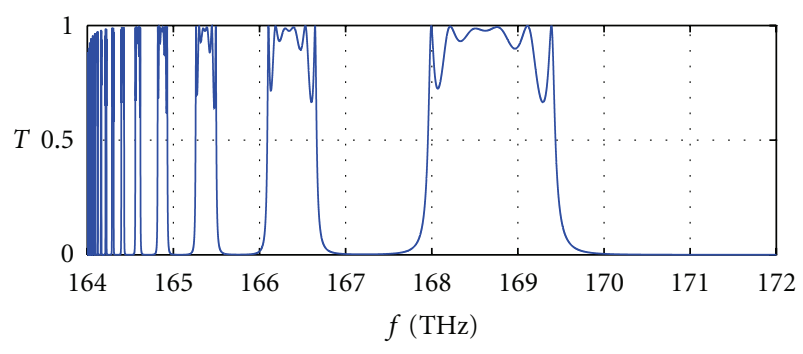

(a)

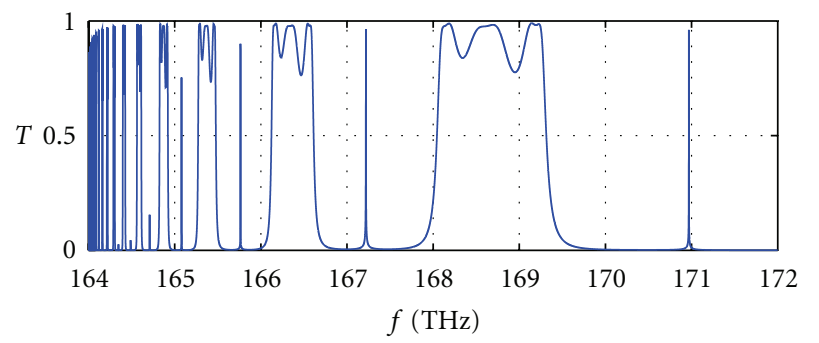

(b)

Figure 3: (Color online) The change of the spectrum shape of transmission coefficient $T$ for alternating 15-layered $\lambda / 4$ spherical stack containing $\mathrm{LH}$ and $\mathrm{SiO}_{2}$ layers (spherical number is $m=1$ ) without and with a defect: (a) strictly periodic case; (b) the stack with a defect LH placed in the center of the stack. The frequency range in both panels corresponds to the frequency range where the real part of LH refraction index is negative; see the inset of Figure 2(b).

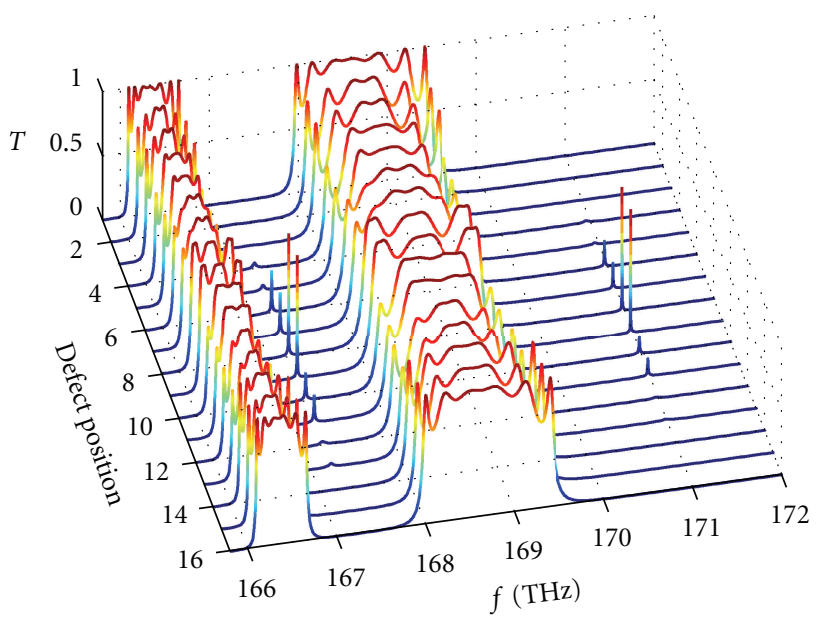

Figure 4: (Color online) Frequency spectrum of the transmission coefficient $T$ for the periodic multilayers microsphere composed by 15 alternating $\lambda / 4$ layers $\mathrm{LH}$ and $\mathrm{SiO}_{2}$ with a $\mathrm{LH}$ defect embedded in various positions in the stack: from 1 to 16 number of layers.

letters $A, B, C, D$ indicate the materials in the spherical stack, $G$ is a defect layer, $\{B, C\}$ indicates the part of spherical stack, respectively. The bottom microsphere has refraction index $n_{4}=1.5+2 \cdot 10^{-4} i(A$, glass, radius $1000 \mathrm{~nm})$. Refraction indices of the LH layers are given by (13), while $n_{C}=1.46+$ $10^{-3} i\left(\mathrm{SiO}_{2}\right.$, width $\left.300 \mathrm{~nm}\right)$ [39] and $n_{D}=1$ ( $D$, surrounding space). To investigate the realistic layer case we have added to

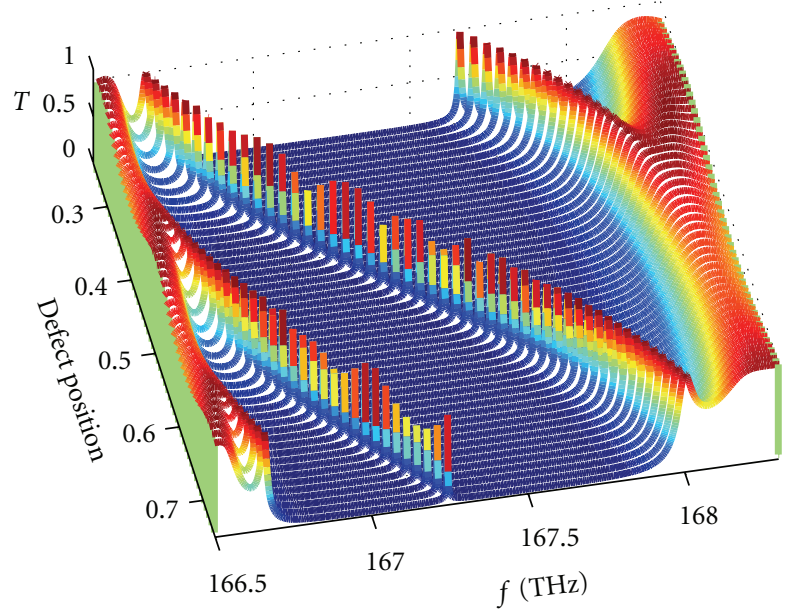

FIGURE 5: (Color online) The frequency spectrum of the transmission coefficient $T$ at the variation of the defect width from $\lambda / 4$ to $3 \lambda / 4$. The multilayered microsphere is composed by 15 alternating layers with $\mathrm{LH}$ and $\mathrm{SiO}_{2}$, and $\mathrm{LH}$ defect is embedded in the center of the stack.

each $n_{i}$ a small imaginary part that corresponds to a material dissipation.

Figure 2 compares the structure of the frequency spectrum of the transmission coefficient for the spherical stack containing conventional materials, Figures 2(a) and 2(c); the case when $\mathrm{Si}$ is replaced by $\mathrm{LH}$ metamaterial is shown in Figures 2(b) and 2(d). In the latter figure it is shown the frequency range where the real part of the refraction index $n(\omega)$ of a metamaterial is negative; see an insert Figure 2(b). We observe that the transmittance spectra in Figures 2(c) and 2 (d) have very different structure. Such a distinction leads to an essential change also in a frequency spectrum of spherical system with a defect layer. Further we mainly study the details of a transmittance spectrum for a spherical system with the LH defect embedded.

Figure 3(a) shows the case of the $\lambda / 4$ spherical stack without a defect, whereas in Figure $3(\mathrm{~b})$ the situation when a LH defect is embedded in the center of the spherical stack is depicted. We observe from Figures 3(a) and 3(b) that the bandgaps become less for frequencies that are closer to the dispersive area with larger values of the refraction index $|n|$. (It is worth noting that in very recent experiment [40] demonstrated that a broadband, extremely high index of refraction can be realized from large-area, free-standing, flexible terahertz metamaterials composed of strongly coupled unit cells.) Besides, from Figure 3(b) we observe that the narrow well-separated resonant peaks with nearly complete transmittance arise in the center of the forbidden frequency zone.

Figure 3 represents only main spectral features of multilayered spherical system with an embedded LH defect. Further we study the details of the transmittance peak with respect to the change of width, position, and the field spherical quantum number. Another important feature considered here is the dependence of the peak details on the random deviation in the widths of the stack layers. 


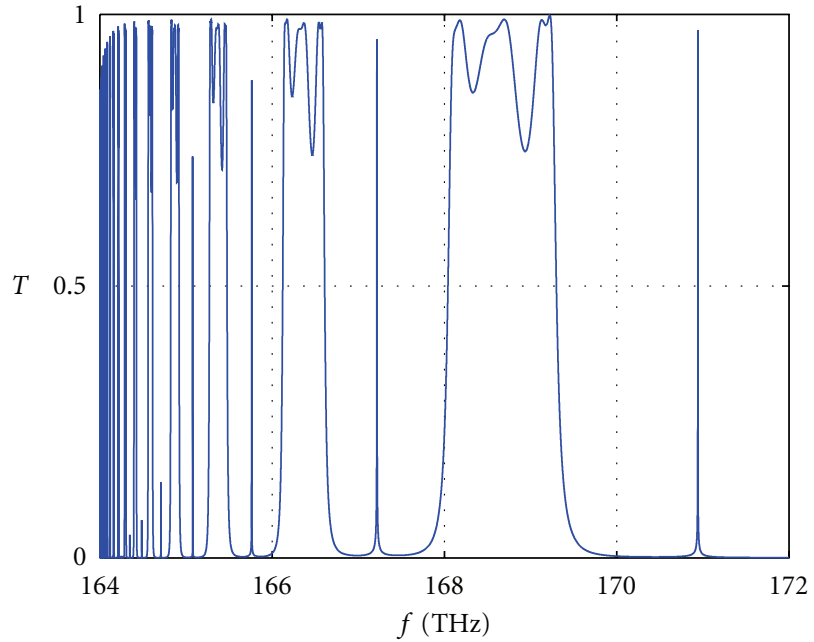

(a)

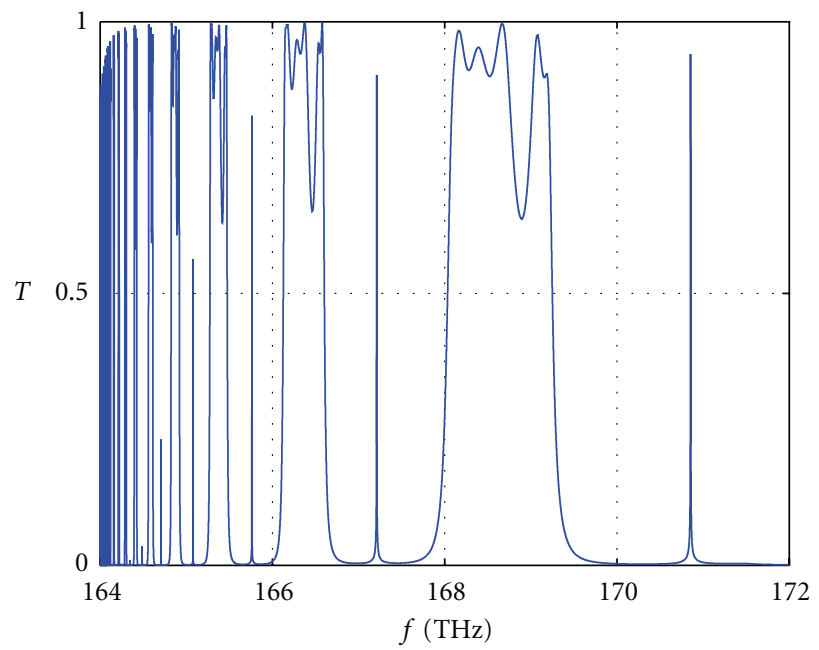

(c)

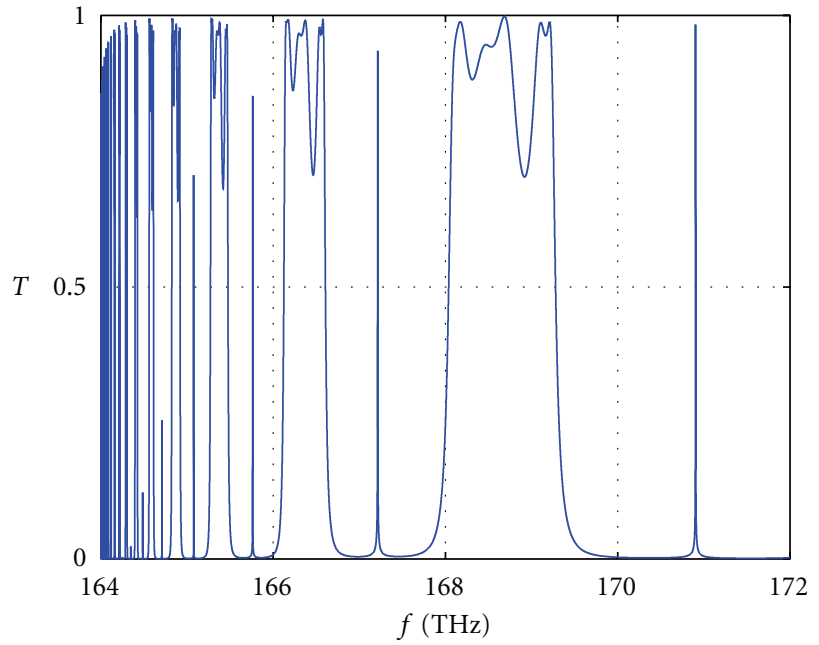

(b)

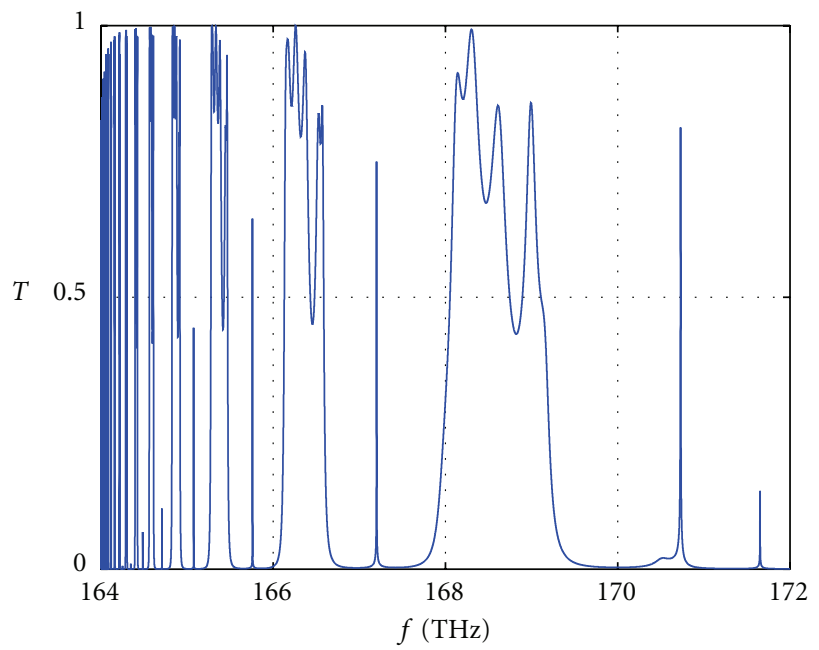

(d)

FIgure 6: (Color online) The dependence of the transmission coefficients $T$ on the spherical quantum numbers $m$ for the periodic multilayers microsphere system composed of 15 alternating layers of $\mathrm{LH}$ and $\mathrm{SiO}_{2}$ and a defect of $\mathrm{LH}$ placed in the center of the system: (a) $m=2$; (b) $m=3$; (c) $m=4$; and (d) $m=6$.

Figure 4 shows the details of transmittance peak in the frequency band gap when a defect is embedded in different positions in the stack. The system consists of 15 alternating $\lambda / 4$ layers $\mathrm{LH}+\mathrm{SiO}_{2}$ and the embedded $\mathrm{LH}$ defect layer. We observe from Figure 4 that the transmittance resonance reaches the maximum value for a case when the defect is placed in center of the stack.

The behavior of transmittance peak in the frequency band gap of the alternating 15 -layered $\lambda / 4$ spherical stack, at variations of the defect layer width (the defect is embedded in the center of the stack, layer 8) is shown in Figure 5. Our calculations have shown that the position of the peak strongly depends on the defect layer width $d$ and does not depend significantly on the material. From Figure 5 we observe that for defect thickness $d=\lambda / 4$ the peak is situated in the center of the band gap, and it migrates to the lower frequencies range when $d$ increases.
In order to investigate the features of such an effect closely to the WGM regime we studied the dependence of the peak width on the spherical quantum number $m$; see Figure 6 . The alternation spherical stack is composed by 15 alternating layers $\mathrm{LH}, \mathrm{SiO}_{2}$, and the $\mathrm{LH}$ defect layer is embedded in the center of the stack. Such a behavior can be easily explained as follows. At a large $m$, there is a transition to the WGM (whispering gallery mode) which occurs when the optical field is exponentially localized near the surface of the microsphere. In this regime, the field does not "feel" the details of the spherical stack structure. Thus, with the increase $m$ the amplitude of the peak will decrease rapidly since at a large $m$, the spatial structure of the layers does not essentially affect the spectrum of the optical oscillations.

Figure 7(a) studies the vicinity of some "defect" resonances with narrow spectral width that have transmittance $T$ close to 1 . On the other hand it is interesting to explore 


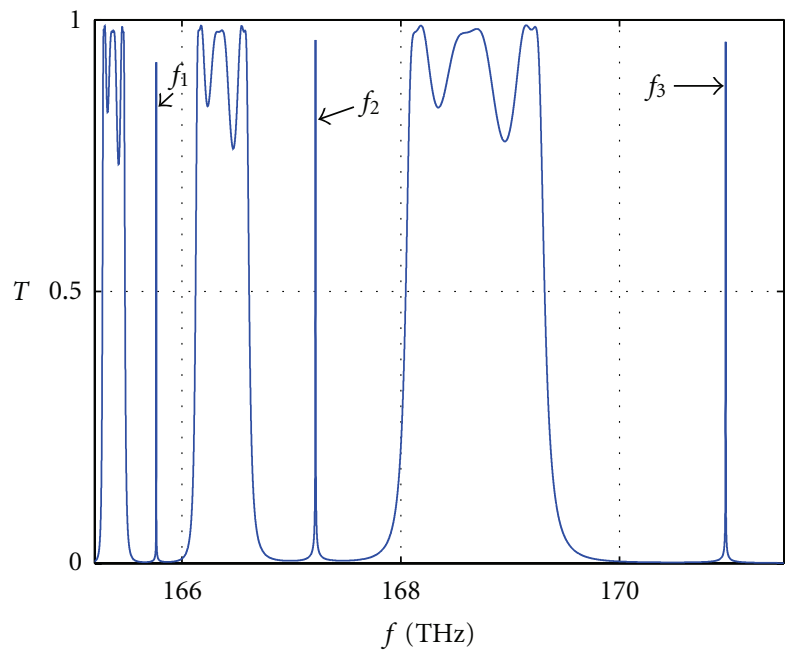

(a)

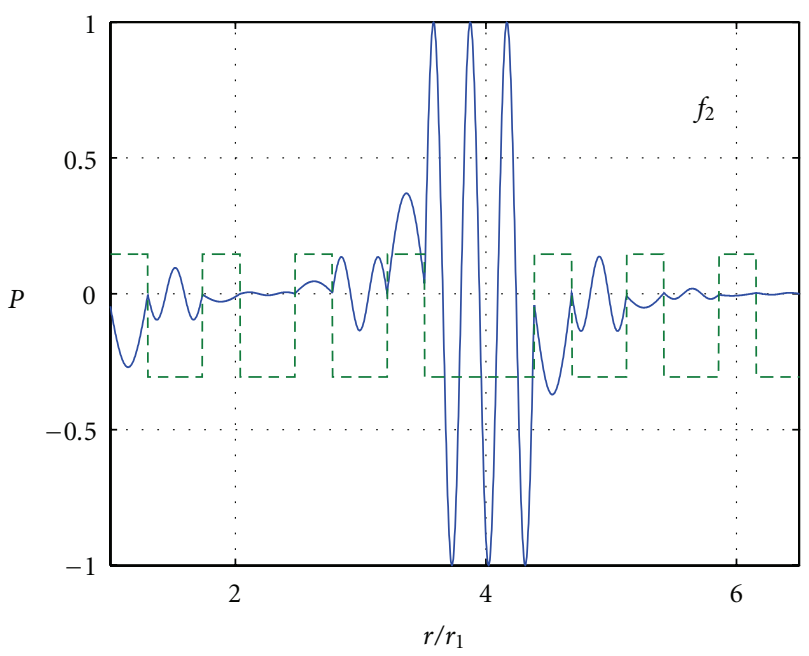

(c)

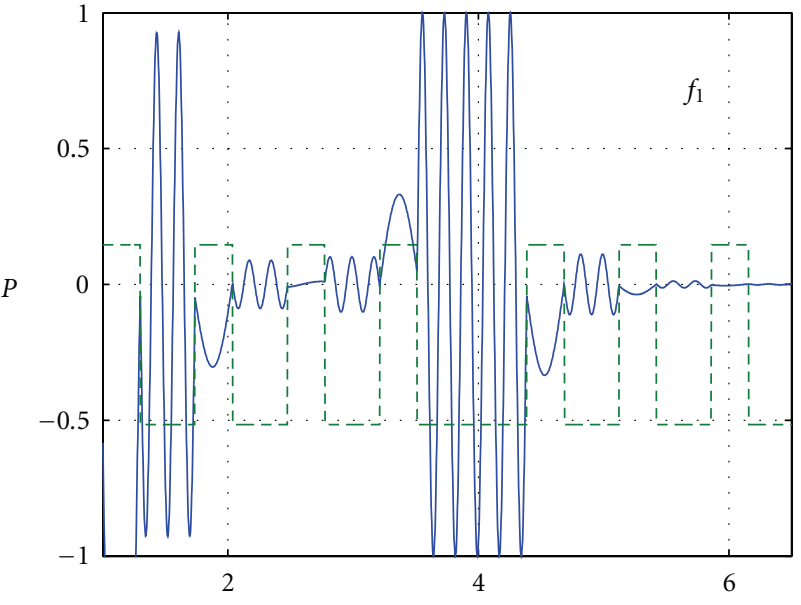

(b)

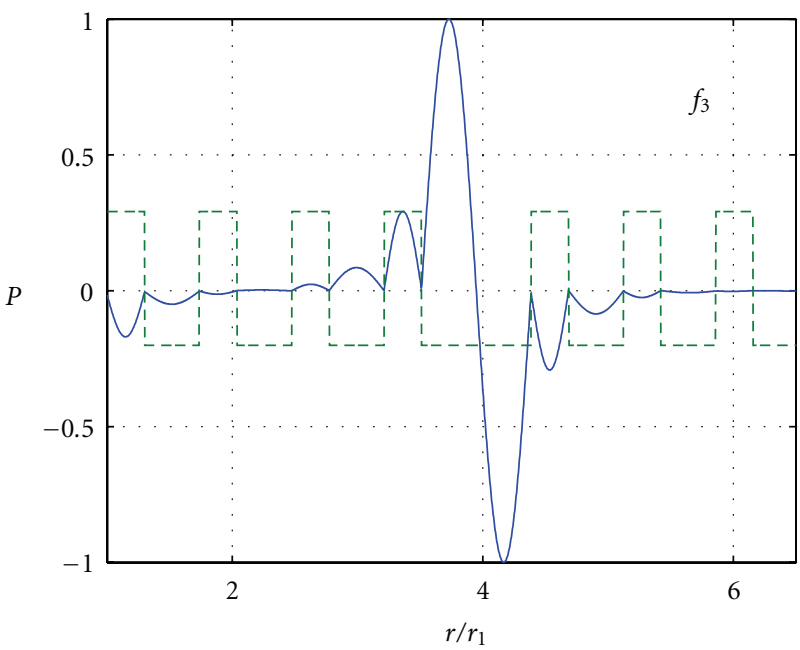

(d)

Figure 7: (Color online) The field structure for various resonances (numerated as $f_{1,2,3}$ ) produced by a defect in the forbidden frequency gaps. (a) shows the spectrum of transmission coefficient $T$ for the periodic multilayers microsphere system composed of 15 alternating layers of $\mathrm{LH}$ and $\mathrm{SiO}_{2}$ and a $\mathrm{LH}$ defect placed in the center of the stack. In (b), (c), and (d) show the radial dependence of the field (arbitrary units) on $r / r_{1}$ corresponding to the resonant peaks marked in (a) as $f_{1}, f_{2}, f_{3}$, respectively. The dashed lines in (b), (c), and (d) show the structures of the spherical stack (refractive indices). The spherical number is $m=1, r_{1}$ is radius of the internal bottom microsphere.

the spatial distribution of the optical field at frequencies of such resonances. Since such a system is very dispersive in the resonant peak area, we calculated the average Poynting vector instead of the average field energy; see Chapter 9 in [41]. The normalized field flux (radial component $(\mathbf{P})_{r}$ of the Poynting vector $\mathbf{P}=[\mathbf{E} \times \mathbf{H}] \rightarrow \mathbf{P} / \mathbf{P}_{\max }$ at the resonant peak frequency $f=\omega / 2 \pi$, solid line), as well as the radial dependence of the refraction indices $n$ of the spherical stack (dash line) in Figures 7(b), 7(c) and 7(d) is shown.

We observe from Figures $7(\mathrm{~b}), 7(\mathrm{c})$, and $7(\mathrm{~d})$ the confinement of the field that is arrested in the area of a LH defect for all resonances. The localization of the defect photon mode can be explained as following [42]. Once a photon enters the defect region, it encounters two $\lambda / 4$ Bragg reflectors (the periodic parts of the stack) before and behind it. This leads to the fact that the photon will be strongly reflected back to the defect region and thus remains long time in the defect area. Such a long dwell time results in very high energy field density around the defect.

In above we have paid more attention to the purely quarter-wave stack case; however, we now study what happens when the thicknesses of the layers in the stack have some random deviations (technological roughness) from the ordered $\lambda / 4$ width. In this case, the far-order periodicity in the system is broken, and each particular layer has an influence on the total properties of the stack.

Figure 8 shows that random deviations in the width layer of the $\lambda / 4$ stack results in the change of the resonance 


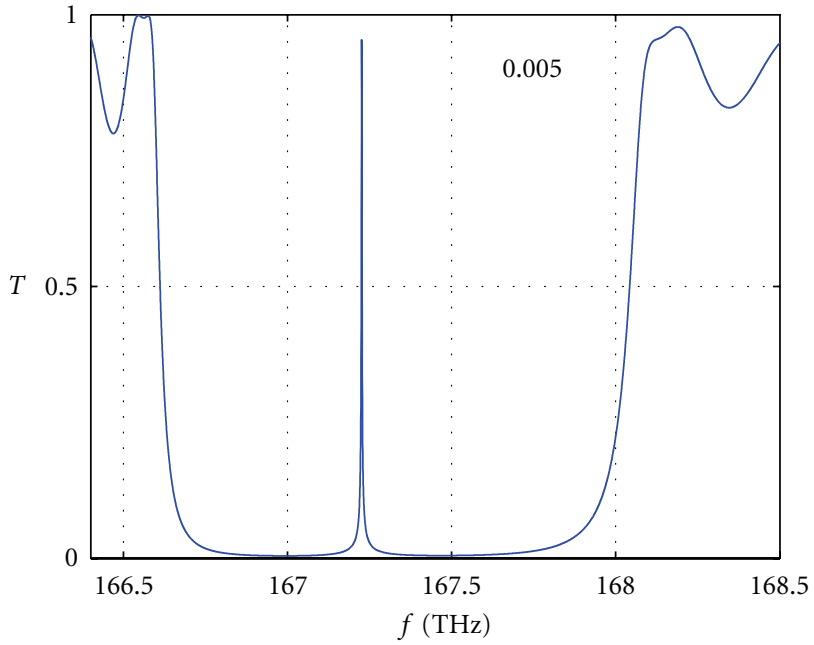

(a)

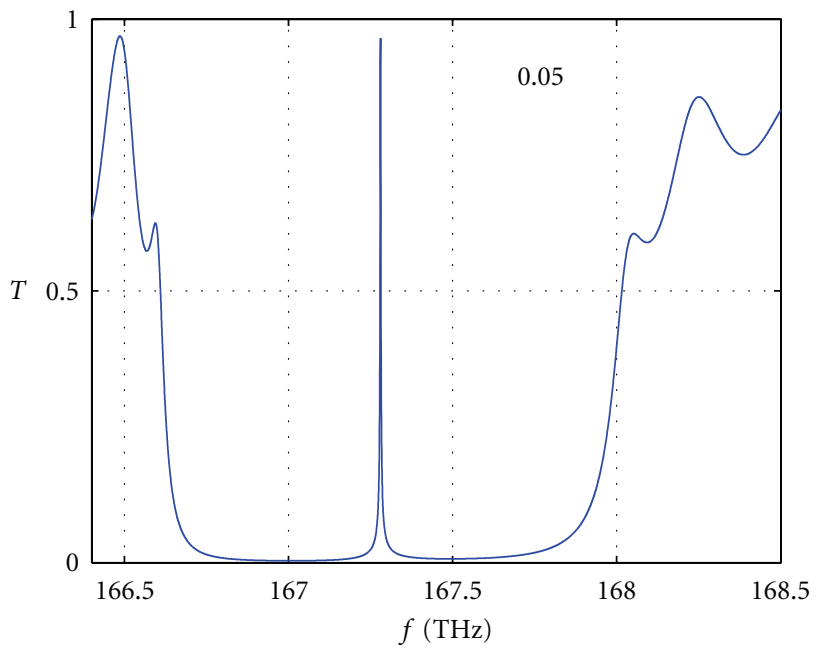

(c)

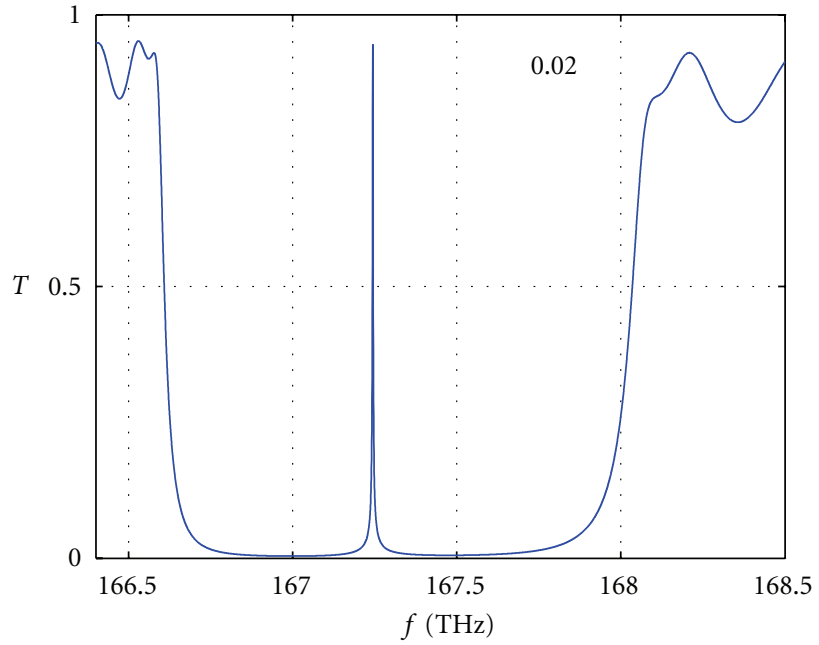

(b)

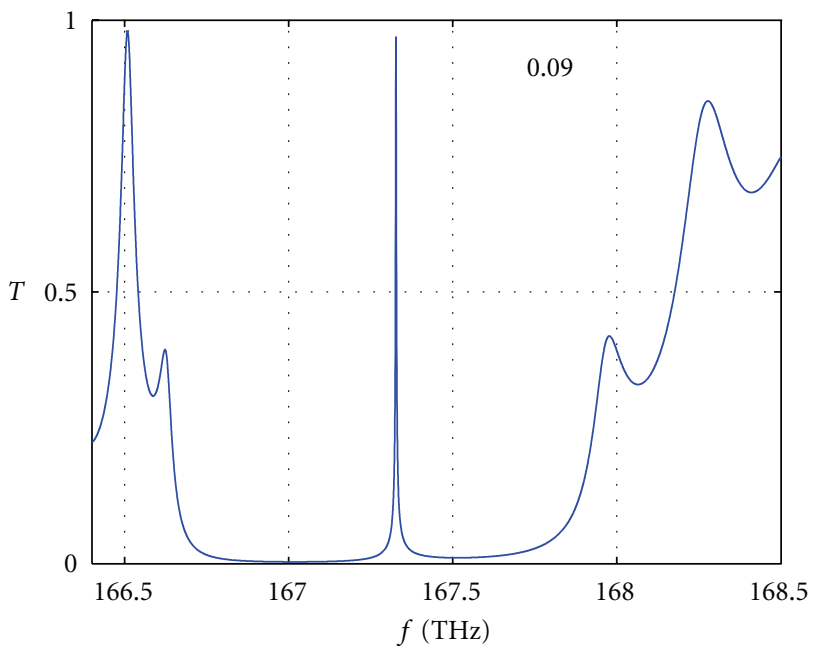

(d)

Figure 8: (Color online.) The transmittance peak position for a microsphere coated by 15 alternating layers and the LH defect embedded in the center for cases: (a) $0.5 \%$, (b) $2 \%$, (c) $5 \%$, and (d) $9 \%$ random deviations in the width of all the layers of the spherical stack from the exact $\lambda / 4$ case.

position. One can see that despite the value of the random deviation level (a) $0.5 \%$, (b) $2 \%$, (c) $3 \%$, and (d) $9 \%$ the peak band remains very narrow. We can explain that as follows. For a range of frequencies in the band gap, the field is confined already in the vicinity of the defect layer and farther is considerably reduced. As a result, the field in the other layers of the stack practically does not affect the structure of the confined field in the defect layer. Such behavior appreciably differs from the case of a stack with strong alternating layers (without defects). In the later case the formation of the spectral peaks is a collective effect due to the wave re-reflections from the great number of individual layers. In such structures small random deviations in a layers' width can be statistically recompensed and result in the destruction of the frequency resonances.
From the above, we can conclude that the transmittance spectrum of spherical structures with an embedded LH defect is rather stable with respect to random deviations. The considered confinement of the field by the defect layer is a typical internal property of such nonuniform metamaterial structures.

\section{Green Function and Spectrum of Nanoemitters Radiation}

In the above we have studied the transmittance properties of the compound spherical stack with a defect, particularly at separated $m$. Other question of fundamental interest is the details of optical spectrum of nanoemitter radiating 


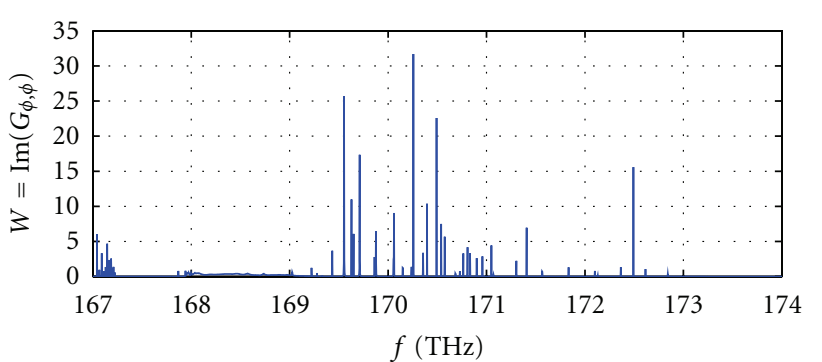

(a)

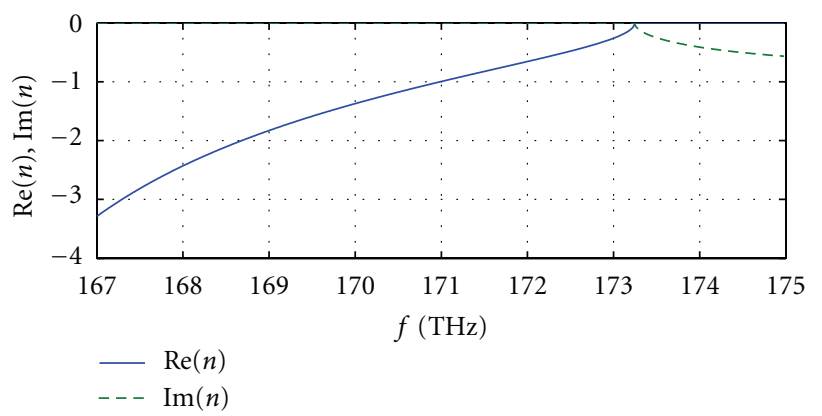

(b)

Figure 9: (Color online) (a) The frequency spectrum of the imaginary part of the Green function $W=\operatorname{Im}\left(G_{\varphi \varphi}(r, r, f)\right),(20)$ (arbitrary units); (b) real and imaginary parts of the LH material refractive index $n$ in the frequency area closely to the metamaterial resonances.

from such a coated microsphere. The latter is formed by a superposition of re-reflecting optical waves with different $m$. Such a study already requires more advanced formalism, namely, the Green function approach. In this section we apply the Green function technique to study the frequency spectrum of a nanoemitter radiation. The spatial scale of the nanoemitter objects $(\sim 1-100 \mathrm{~nm})$ is at least one order of magnitude smaller than the spatial scale of microspheres $\left(\sim 10^{3}-10^{4} \mathrm{~nm}\right)$. Therefore in the coated microsphere (Figure 1), we can represent the nanoemitter structure as a point source placed at $\mathbf{r}^{\prime}$ and having a dipole moment $\mathbf{d}_{0}$. It is well known that the solution of the wave equation for the radiated electromagnetic field $\mathbf{E}$ due to a general source $\mathbf{J}\left(\mathbf{r}^{\prime}\right)$ is $[33,43,44]$

$$
\mathbf{E}(\mathbf{r})=i \omega \mu_{0} \mu \int_{V} d \mathbf{r}^{\prime} \widehat{\mathbf{G}}\left(\mathbf{r}, \mathbf{r}^{\prime}, \omega\right) \cdot \mathbf{J}\left(\mathbf{r}^{\prime}\right)
$$

where $\hat{\mathbf{G}}\left(\mathbf{r}, \mathbf{r}^{\prime}, \omega\right)$ is the dyadic Green function (DGF), which depends on the type of boundary conditions imposed on $\mathbf{E}(\mathbf{r})$ and contains all the physical information necessary for describing the multilayered structure (the time dependence is assumed to be $e^{i \omega t}$ ). Equation (16) is complemented by the standard boundary conditions: limitation of the fields in the center of the microsphere and continuity of the tangential components of the fields at the interfaces of layers. We also use Sommerfeld's radiation conditions, where there is only an outgoing wave in the external boundary of the microsphere. In this case, the electromagnetic field $\mathbf{E}$ in the coated structure consists of the sum of the waves radiating in the surrounding medium and the multiple wave reflections due to the interfaces between layers. Substituting the nanoemitter source in the form $\mathbf{J}=i \omega \mathbf{d}, \mathbf{d}=\mathbf{d}_{0} \delta\left(\mathbf{r}-\mathbf{r}^{\prime}\right)$ in (16), we obtain

$$
\mathbf{E}\left(\mathbf{r}, \mathbf{r}^{\prime}, \omega\right)=-\mathbf{p}_{0} \hat{\mathbf{G}}\left(\mathbf{r}, \mathbf{r}^{\prime}, \omega\right)
$$

where $\mathbf{p}_{0}=\left(\mu \mathbf{d}_{0} / \varepsilon_{0}\right)\left(\omega^{2} / c^{2}\right)$. In such a situation, the nanoemitter frequency spectrum is identical to the dyadic Green's function (DGF) spectrum. Thus, the equation of the field generated by a nanoemitter assumes the form of the DGF $\mathbf{G}\left(\mathbf{r}, \mathbf{r}^{\prime}, \omega\right)$ equation and is given by $[43,44]$

$$
\left[(\nabla \times \kappa(\mathbf{r}, \omega)) \times \nabla-\frac{\omega^{2}}{c^{2}} \varepsilon(\mathbf{r}, \omega)\right] \hat{\mathbf{G}}\left(\mathbf{r}, \mathbf{r}^{\prime}, \omega\right)=\delta\left(\mathbf{r}-\mathbf{r}^{\prime}\right) \hat{\mathbf{I}}
$$

where $\kappa(\mathbf{r}, \omega)=\mu^{-1}(\mathbf{r}, \omega) ; \mathbf{r}$ is the point where the field is observed, while $\mathbf{r}^{\prime}$ is the nanoemitter (point source) location. The dyadic Green's function $\hat{\mathbf{G}}\left(\mathbf{r}, \mathbf{r}^{\prime}, \omega\right)$ also satisfies the boundary conditions at the interfaces of spherical layers $[43,45]$

$$
\begin{aligned}
\hat{\mathbf{r}} \times \hat{\mathbf{G}}^{f s} & =\hat{\mathbf{r}} \times \widehat{\mathbf{G}}^{f+1, s}, \\
\kappa_{f}(\mathbf{r}, \omega) \times \hat{\mathbf{r}} \times \widehat{\mathbf{G}}^{f s} & =\kappa_{f+1}(\mathbf{r}, \omega) \times \widehat{\mathbf{r}} \times \widehat{\mathbf{G}}^{f s} .
\end{aligned}
$$

Let us consider the multilayered spherical structure: a concentric system of spherical layers contacting with the sphere (concentric stack) deposited onto the surface of the microsphere with nanoemitters placed in such a structure (see Figure 1). The layers are localized at the distance $R_{k}$ from the center, where $d_{k}=R_{k}-R_{k+1}$ is the width of a $k$ th layer.

Let us first specify some details of the Green's function technique for multilayered microspheres and introduce our notations. Following the approach [45], we write down DGF of such a system as follows:

$$
\hat{\mathbf{G}}_{e}^{f s}\left(\mathbf{r}, \mathbf{r}^{\prime}, \omega\right)=\hat{\mathbf{G}}_{0 e}^{V}\left(\mathbf{r}, \mathbf{r}^{\prime}, \omega\right) \delta_{f s}+\hat{\mathbf{G}}_{e s}^{(f s)}\left(\mathbf{r}, \mathbf{r}^{\prime}, \omega\right)
$$

where $\hat{\mathbf{G}}_{0 e}^{V}$ represents the contribution of the direct waves from the radiation sources in the unbounded medium, whereas $\hat{\mathbf{G}}_{e s}^{(f s)}$ describes the contribution of the multiple reflection and transmission waves due to the layer interfaces. The dyadic Green's tensor $\widehat{\mathbf{G}}_{0 e}^{V}\left(\mathbf{r}, \mathbf{r}^{\prime}, \omega\right)$ in $(20)$ is given by

$$
\begin{aligned}
\widehat{\mathbf{G}}_{0 e}^{V}\left(\mathbf{r}, \mathbf{r}^{\prime}, \omega\right)= & \frac{\widehat{\mathbf{r}} \hat{\mathbf{r}}}{k_{s}^{2}} \delta\left(\mathbf{r}-\mathbf{r}^{\prime}\right) \\
& +\frac{i k_{s}}{4 \pi} \sum_{q=e, o} \sum_{m=1}^{\infty} \sum_{l=0}^{m} C_{n m} \widehat{\mathbf{G}}_{q, m l}^{V}\left(\mathbf{r}, \mathbf{r}^{\prime}, \omega\right),
\end{aligned}
$$

where tensor $\mathbf{G}_{q, n m}^{V}\left(\mathbf{r}, \mathbf{r}^{\prime}, \omega\right)$ in $(21)$ has a form 


$$
\hat{\mathbf{G}}_{q, n m}^{V}\left(\mathbf{r}, \mathbf{r}^{\prime}, \omega\right)=\left(\begin{array}{l}
\mathbf{M}_{q, n m}^{(1)}\left(\mathbf{r}, k_{s}\right) \mathbf{M}_{q, n m}\left(\mathbf{r}^{\prime}, k_{s}\right)+\mathbf{N}_{q, n m}^{(1)}\left(\mathbf{r}, k_{s}\right) \mathbf{N}_{q, n m}\left(\mathbf{r}^{\prime}, k_{s}\right), \mathbf{r}>\mathbf{r}^{\prime} \\
\mathbf{M}_{q, n m}\left(\mathbf{r}, k_{s}\right) \mathbf{M}_{q, n m}^{(1)}\left(\mathbf{r}^{\prime}, k_{s}\right)+\mathbf{N}_{q, n m}\left(\mathbf{r}, k_{1}\right) \mathbf{N}_{q, n m}^{(1)}\left(\mathbf{r}^{\prime}, k_{s}\right), \mathbf{r}<\mathbf{r}^{\prime}
\end{array}\right) .
$$

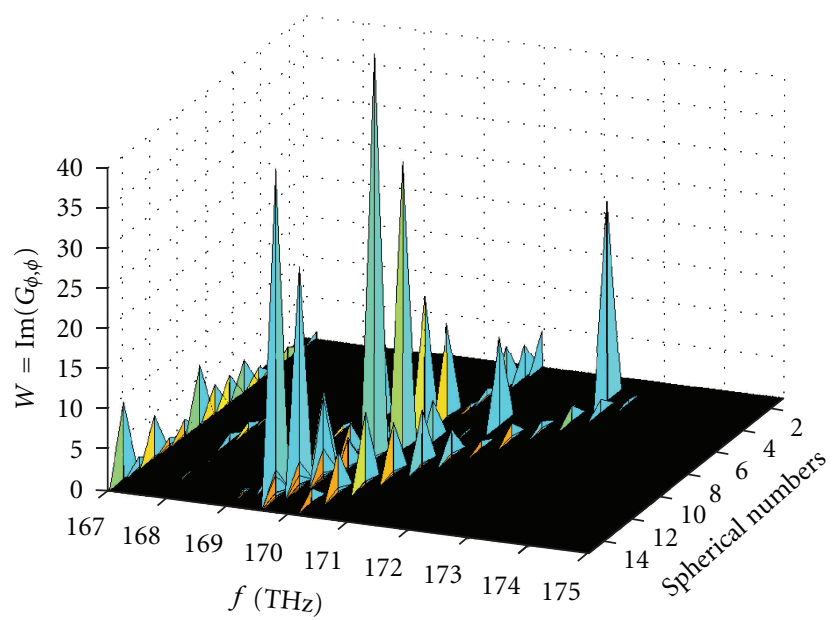

FIGURE 10: (Color online) The frequency spectrum of the imaginary part of the Green function $W=\operatorname{Im}\left(G_{\varphi \varphi}(r, r, f)\right)$ (arbitrary units) for partial spherical quantum numbers $m$ in the area closely to the metamaterial resonances.

In (22), vectors $\mathbf{M}$ and $\mathbf{N}$ represent TE- and TM-waves, where the prime denotes the nanoemitter coordinates $\left(r^{\prime}, \theta^{\prime}, \varphi^{\prime}\right)$, $n$ and $m$ are spherical and azimuthal quantum numbers, respectively, while $k_{s}$ is the wave number of the medium where the radiated nanoemitters are located. It is worth noting that due to the dyad $\widehat{\mathbf{r}} \hat{\mathbf{r}}$, the $\delta$-function in (21) contributes to the radial (longitudinal) part [43]. Due to the equality $\hat{\mathbf{r}} \cdot(\theta \hat{\theta}+\varphi \hat{\varphi})=0$ (where $\hat{\mathbf{r}}, \hat{\theta}$, and $\hat{\varphi}$ are the unitary vectors of the spherical coordinates) such a singularity does not contribute to the field (17) for case of the tangential dipole. Since nanoemitters (nanorods) are highly polarized objects, we pay more attention to the case when the dipole orientation of nanoemitter is $\mathbf{d}=d \hat{\varphi}$, so only the tangential components of the Green's tensor $\hat{G}_{\varphi \varphi}$ contribute. Description of the Green function approach and complete expression for the scattering Green tensor $\widehat{\mathbf{G}}_{e s}^{(f s)}\left(\mathbf{r}, \mathbf{r}^{\prime}, \omega\right)$ is rather bulky and can be found in [22, 28, 45].

It is well known that due to the fluctuation-dissipation theorem the correlation function of the photon states in the absorbing environment for temperature $T$ can be written down with the help of the macroscopic Green-function as follows [46]:

$$
\left\langle\mathbf{E}(r) \mathbf{E}\left(r^{\prime}\right)\right\rangle_{\omega}=\frac{\hbar \omega^{2}}{c^{2}} \operatorname{coth}\left(\frac{\hbar \omega}{2 T}\right) \operatorname{Im}\left(\widehat{\mathbf{G}}\left(r, r^{\prime}, \omega\right)\right) .
$$

In particular, from (23) one can see that the case $r=r^{\prime}$ yields the inequality $\operatorname{Im}(\widehat{\mathbf{G}}(r, r))>0$ that corresponds to the energy of a fluctuating electromagnetic field $\mathbf{E}(\mathbf{r})$ at small dissipation [46]: $\left\langle\mathbf{E}(\mathbf{r})^{2}\right\rangle \sim \operatorname{Im}(\widehat{\mathbf{G}}(\mathbf{r}, \mathbf{r}))$, where $\widehat{\mathbf{G}}(\mathbf{r}, \mathbf{r})$ is the Green function, that is, $\hat{\mathbf{G}}_{\varphi \varphi}(\mathbf{r}, \mathbf{r})$ in our case. The typical structure of the Green function spectrum is shown in Figure 9(a). We observe various peak frequency resonances in the area where real part of the LH material is negative $\operatorname{Re}(n)<0$ (see Figure 9(b)). However no resonances one observes in the frequency zone $f \geq 173 \mathrm{THz}$, where $\operatorname{Re}(n) \geq 0$.

In general a number of spherical harmonics contribute to the spectrum of the emitter's radiation in a microsphere. We investigate the frequency distribution of the field energy $W=\operatorname{Im}\left(\hat{\mathbf{G}}_{\varphi \varphi}(r, r, f)\right)$ at partial spherical numbers $m$ in order to see the transit to the WGM regime. In Figure 10 the structure of $W$ peaks at different spherical numbers $m$, in a vicinity of the metamaterial resonances, is shown. The multilayered spherical stack is composed by 12 alternating layers $\mathrm{LH}, \mathrm{SiO}_{2}$, and the $\mathrm{LH}$ defect layer is embedded in the center of the stack. We observe from Figure 10 the existence of a strong frequency peak in area $f \sim 170.5 \mathrm{THz}$. However, the number of such peaks decreases for larger $m \gg 1$ that corresponds to the transit to the WGM regime. Such a behavior can be explained as follows. At large $m$ (that is an index of the spherical Bessel function $j_{m}(z)$ in DGF) the following asymptotic expression takes place [47] $j_{m}(z) \simeq$ $(2 \pi m)^{-0.5}(e z)^{m} / 2 m$. Thus, at some $z=r / r_{\text {ext }}<1\left(r_{\text {ext }}\right.$ is the external radius) the optical amplitudes will decrease rapidly for deeper $r \ll r_{\text {ext }}$ and large $m$. This results in concentration of the photonic field closely to the external boundary of microsphere; therefore field does not "feel" the details of multilayered spherical structure. This leads to a diminution of the boundary re-reflections and phase interferences, which normally are observed as frequency resonant peaks.

\section{Conclusion}

We have investigated optical transmission through a compound spherical stack with conventional and metamaterial (MM) layers and also embedded MM defect. We have found that incorporation of such a defect allows a formation of extremely narrow resonant peaks with nearly complete transmittance in area of a band gap. It is found a strong dependence of the spectral position of the resonance on the width of the defect layer. The latter in principle opens new possibilities creating optical filters with extremely narrow passbands in the desired frequency range. We demonstrate that the optical fields of certain frequencies can be arrested by a left-handed (LH) defect. The latter can be used to obtain resonant field structures by engineering defects in microspheres with a compound conventional and metamaterial dielectric stack. 


\section{References}

[1] V. G. Veselago, "The electrodynamics of substances with simultaneously negative values of $\varepsilon$ and $\mu$," Soviet PhysicsUspekhi, vol. 10, pp. 509-514, 1968.

[2] J. B. Pendry, A. J. Holden, D. J. Robbins, and W. J. Stewart, "Magnetism from conductors and enhanced nonlinear phenomena," IEEE Transactions on Microwave Theory and Techniques, vol. 47, no. 11, pp. 2075-2084, 1999.

[3] D. R. Smith, W. J. Padilla, D. C. Vier, S. C. Nemat-Nasser, and S. Schultz, "Composite medium with simultaneously negative permeability and permittivity," Physical Review Letters, vol. 84, no. 18, pp. 4184-4187, 2000.

[4] I. I. Smolyaninov, Y. J. Hung, and C. C. Davis, "Magnifying superlens in the visible frequency range," Science, vol. 315, no. 5819, pp. 1699-1701, 2007.

[5] V. M. Shalaev, "Optical negative-index metamaterials," Nature Photonics, vol. 1, no. 1, pp. 41-48, 2007.

[6] S. Xiao, V. P. Drachev, A. V. Kildishev et al., "Loss-free and active optical negative-index metamaterials," Nature, vol. 466, no. 7307, pp. 735-738, 2010.

[7] V. B. Braginsky, M. L. Gorodetsky, and V. S. Ilchenko, "Quality-factor and nonlinear properties of optical whispering-gallery modes," Physics Letters A, vol. 137, no. 7-8, pp. 393 397, 1989.

[8] V. V. Vassiliev, V. L. Velichansky, V. S. Ilchenko, M. L. Gorodetsky, L. Hollberg, and A. V. Yarovitsky, "Narrow-linewidth diode laser with a high-Q microsphere resonator," Optics Communications, vol. 158, no. 1-6, pp. 305-312, 1998.

[9] J. R. Buck and H. J. Kimble, "Optimal sizes of dielectric microspheres for cavity QED with strong coupling," Physical Review A, vol. 67, no. 3, Article ID 033806, 11 pages, 2003.

[10] V. Astratov, Fundamentals and Applications of Microsphere Resonator Circuits in Photonic Microresonator Research and Applications, vol. 156 of Springer Series in Optical Sciences, Springer, 2010.

[11] A. Stratton, Electromagnetic Theory, McGraw-Hill, New York, NY, USA, 1941.

[12] D. Brady, G. Papen, and J. E. Sipe, "Spherical distributed dielectric resonators," Journal of the Optical Society of America $B$, vol. 10, no. 4, pp. 646-657, 1993.

[13] K. G. Sullivan and D. G. Hall, "Radiation in spherically symmetric structures. I. The coupled-amplitude equations for vector spherical waves," Physical Review A, vol. 50, no. 3, pp. 2701-2707, 1994.

[14] G. Burlak, S. Koshevaya, J. Sanchez-Mondragon, and V. Grimalsky, "Electromagnetic oscillations in a multilayer spherical stack," Optics Communications, vol. 180, no. 1, pp. 49-58, 2000.

[15] C. T. Chan, W. Y. Zhang, Z. L. Wang et al., "Photonic band gaps from metallo-dielectric spheres," Physica B, vol. 279, no. 1-3, pp. 150-154, 2000.

[16] H. T. Miyazaki, H. Miyazaki, K. Ohtaka, and T. Sato, "Photonic band in two-dimensional lattices of micrometersized spheres mechanically arranged under a scanning electron microscope," Journal of Applied Physics, vol. 87, no. 10, pp. 7152-7158, 2000.

[17] I. Gourevich, L. M. Field, Z. Wei et al., "Polymer multilayer particles: a route to spherical dielectric resonators," Macromolecules, vol. 39, no. 4, pp. 1449-1454, 2006.

[18] A. Petukhova, A. S. Paton, Z. Wei et al., "Polymer multilayer microspheres loaded with semiconductor quantum dots," Advanced Functional Materials, vol. 18, no. 13, pp. 1961-1968, 2008.
[19] G. N. Burlak, "Enhanced optical fields in a multilayered microsphere with a quasiperiodic spherical stack," Physica Scripta, vol. 76, no. 5, pp. 571-576, 2007.

[20] G. N. Burlak and A. Díaz-de-Anda, "Optical fields in a multilayered microsphere with a quasiperiodic spherical stack," Optics Communications, vol. 281, no. 1, pp. 181-189, 2008.

[21] G. N. Burlak and A. Diaz-De-Anda, "The subwavelength optical field confinement in a multilayered microsphere with quasiperiodic spherical stack," Advances in OptoElectronics, vol. 2008, Article ID 781524, 6 pages, 2008.

[22] G. Burlak, A. Díaz-De-Anda, R. S. Salgado, and J. P. Ortega, "Narrow transmittance peaks in a multilayered microsphere with a quasiperiodic left-handed stack," Optics Communications, vol. 283, no. 19, pp. 3569-3577, 2010.

[23] S. Mahmoodian, R. C. McPhedran, C. M. De Sterke, K. B. Dossou, C. G. Poulton, and L. C. Botten, "Single and coupled degenerate defect modes in two-dimensional photonic crystal band gaps," Physical Review A, vol. 79, no. 1, Article ID 013814, 2009.

[24] V. C. Nguyen, L. Chen, and K. Halterman, "Total transmission and total reflection by zero index metamaterials with defects," Physical Review Letters, vol. 105, no. 23, Article ID 233908, 2010.

[25] G. Burlak, A. Zamudio-Lara, and D. Juarez, "Confinement of electromagnetic oscillations in a dielectric microsphere coated by the frequency dispersive multilayers," Physics Letters A, vol. 289, no. 1-2, pp. 99-105, 2001.

[26] G. N. Burlak, "Optical radiation from coated microsphere with active core," Physics Letters A, vol. 299, no. 1, pp. 94-101, 2002.

[27] A. Moroz, "Photonic crystals of coated metallic spheres," Europhysics Letters, vol. 50, no. 4, pp. 466-472, 2000.

[28] G. Burlak and V. Grimalsky, "High quality electromagnetic oscillations in inhomogeneous coated microsphere," Optics Communications, vol. 263, no. 2, pp. 342-349, 2006.

[29] G. Burlak, The Classical and Quantum Dynamics of the Multispherical Nanostructures, Imperial College Press, 2004.

[30] A. Moroz, "A recursive transfer-matrix solution for a dipole radiating inside and outside a stratified sphere," Annals of Physics, vol. 315, no. 2, pp. 352-418, 2005.

[31] W. Panofsky and M. Phillips, Classical Electricity, AddisonWesley, 1962.

[32] M. Born and E. Wolf, Principles of Optics, Pergamon, New York, NY, USA, 1980.

[33] H. T. Dung, S. Y. Buhmann, L. Knöll, D. G. Welsch, S. Scheel, and J. Kästel, "Electromagnetic-field quantization and spontaneous decay in left-handed media," Physical Review A, vol. 68, no. 4, Article ID 043816, 15 pages, 2003.

[34] R. W. Ziolkowski and E. Heyman, "Wave propagation in media having negative permittivity and permeability," Physical Review E, vol. 64, no. 5, Article ID 056625, 15 pages, 2001.

[35] R. A. Shelby, D. R. Smith, and S. Schultz, "Experimental verification of a negative index of refraction," Science, vol. 292, no. 5514, pp. 77-79, 2001.

[36] N. Garcia and M. Nieto-Vesperinas, "Is there an experimental verification of a negative index of refraction yet?" Optics Letters, vol. 27, no. 11, pp. 885-887, 2002.

[37] P. Kinsler and M. W. McCall, "Causality-based criteria for a negative refractive index must be used with care," Physical Review Letters, vol. 101, no. 16, Article ID 167401, 2008.

[38] M. I. Stockman, "Criterion for negative refraction with low optical losses from a fundamental principle of causality," Physical Review Letters, vol. 98, no. 17, Article ID 177404, 2007. 
[39] S. Ajith Kumar, C. L. Nagendra, H. Ganesh Shanbhogue, and G. K. M. Thutupalli, "Near-infrared bandpass filters from $\mathrm{Si} / \mathrm{SiO} 2$ multilayer coatings," Optical Engineering, vol. 38, no. 2, pp. 368-380, 1999.

[40] M. Choi, S. H. Lee, Y. Kim et al., "A terahertz metamaterial with unnaturally high refractive index," Nature, vol. 470, no. 7334, pp. 369-373, 2011.

[41] L. D. Landau, L. P. Pitaevskii, and E. M. Lifshitz, Electrodynamics of Continuous Media, Butterworth-Heinemann, 2nd edition, 1984.

[42] S. Y. Zhu, N. H. Liu, H. Zheng, and H. Chen, "Time delay of light propagation through defect modes of one-dimensional photonic band-gap structures," Optics Communications, vol. 174, no. 1-4, pp. 139-144, 2000.

[43] W. Chew, Waves, Fields in Inhomogeneous Media, IEEE Press, New York, NY, USA, 1996.

[44] J. D. Jackson, Classical Electrodynamics, John Willey and Sons, New York, NY, USA, 1975.

[45] L. W. Li, P. S. Kooi, M. S. Leong, and T. S. Yeo, "Electromagnetic dyadic Green's function in spherically multilayered media," IEEE Transactions on Microwave Theory and Techniques, vol. 42, no. 12, pp. 2302-2310, 1994.

[46] L. D. Landau and E. M. Lifschitz, Statistical Physics, Part 2, Pergamon Press, Oxford, UK, 1981.

[47] M. Abramowitz and I. A. Stegun, Handbook of Mathematical Functions: With Formulas, Graphs, and Mathematical Tables, Dover Publications, 1965. 

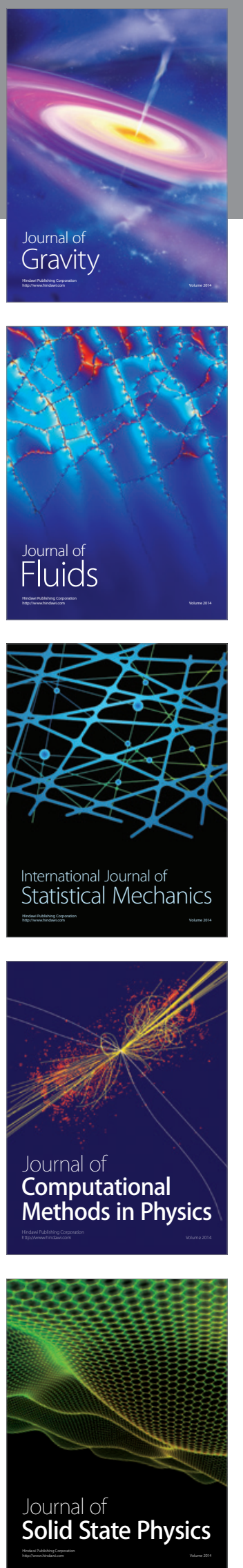

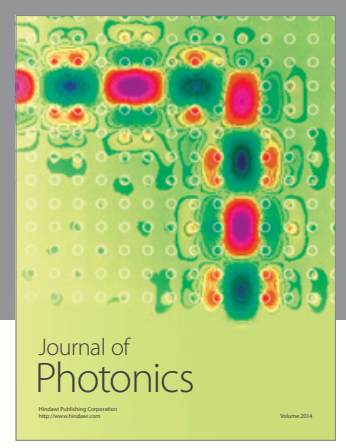

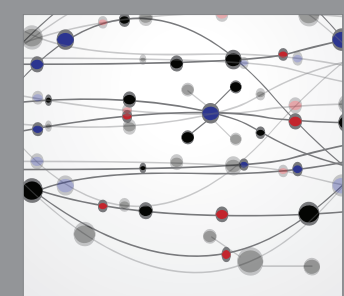

The Scientific World Journal
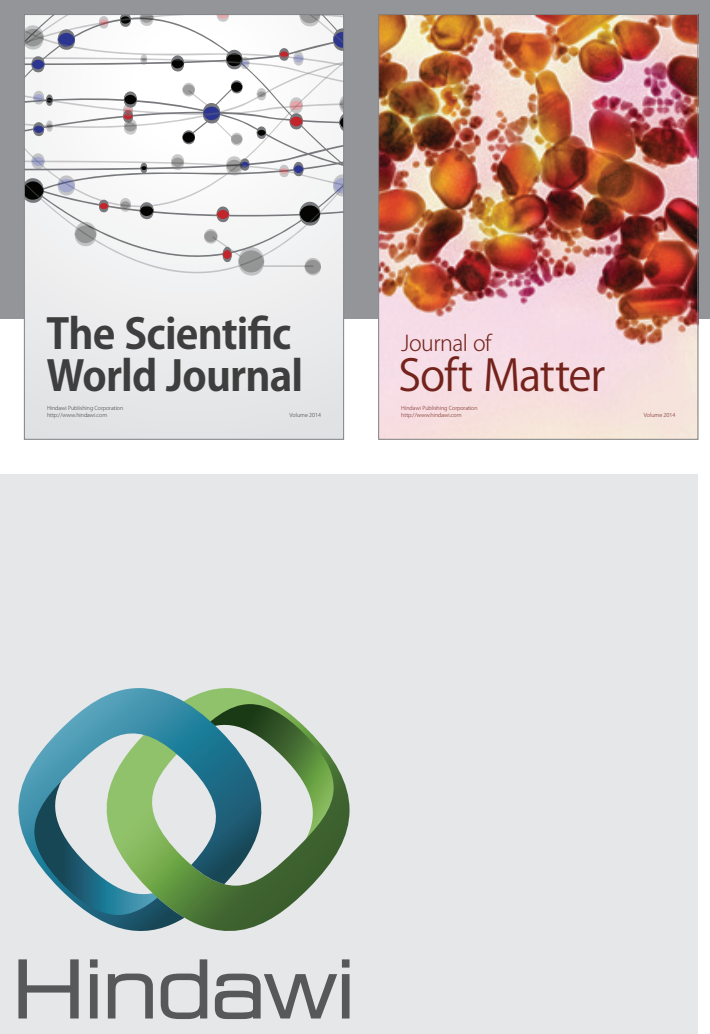

Submit your manuscripts at

http://www.hindawi.com
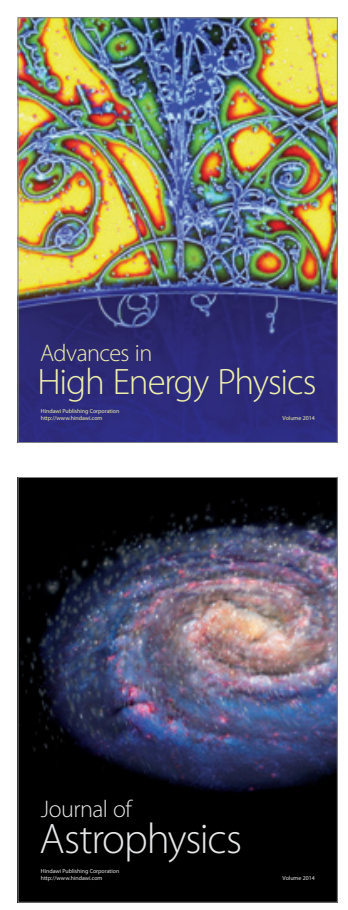
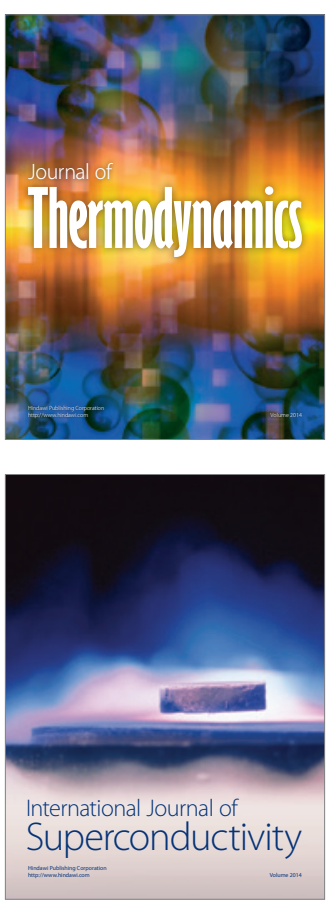
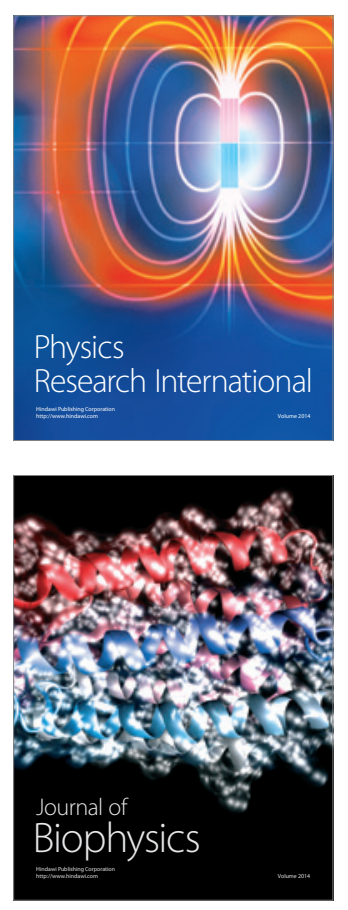
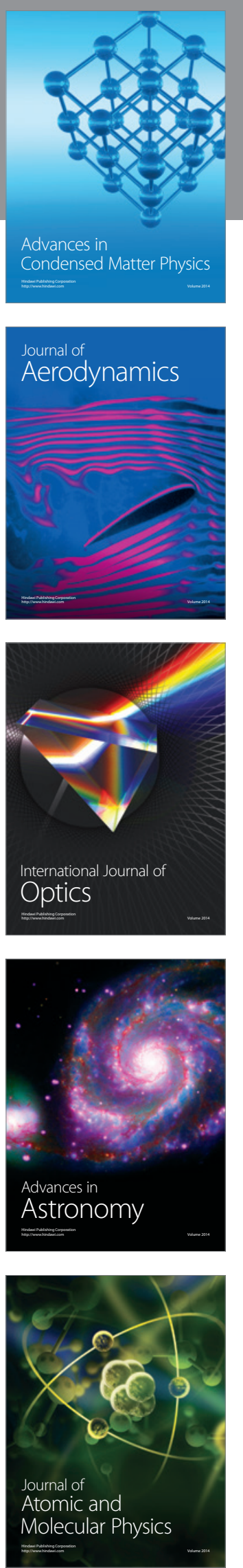